\title{
Integration of disparate data sources to perform maintenance prognosis and optimal decision making
}

\author{
Adriaan Van Horenbeek \& Liliane Pintelon \\ Centre for Industrial Management \\ KU Leuven \\ Leuven, 3001, Belgium \\ Tel. +32 16322497 \\ Fax +32 16322986 \\ Adriaan.VanHorenbeek@cib.kuleuven.be
}

\author{
Diego Galar \\ Division of Operation and Maintenance Engineering \\ Luleå University of Technology \\ Luleå, Sweden
}

\begin{abstract}
Prognosis can be defined as the course of predicting a failure of equipment or a component in advance, whereas prognostication refers to the act of prediction. The three main branches of condition based maintenance are diagnosis, prognosis, and treatmentprognosis, however prognosis is admittedly the most difficult. Also, this area has been the least described in literature and the knowledge about it in a maintenance management context is still poorly systematized. To this day, formal professional attention to prognosis, in the field of maintenance management and engineering in the everyday care of machinery, is often relegated to a secondary status although the availability of prognostic information can considerably improve (e.g. reduce costs, maximize uptime) the performance of machinery and maintenance processes.
\end{abstract}

Ideally, assessment of a prognosis of remaining useful life should be deliberate and explicit. In order to support the maintenance crew in the achievement of this objective an increasing amount of prognostic information is available. Over the last decade, system integration has grown in popularity as it allows organizations to streamline business processes. It is necessary to integrate management data from CMMS (Computer Maintenance Management Systems) with CM (Condition Monitoring) systems and finally SCADA (Supervisory Control And Data Acquisition) and other control systems, widely used in production but with a seldom usage in asset diagnosis and prognosis. The most obvious obstacle in the integration of these data is the disparate nature of the data types involved, moreover several attempts to remedy this problem have fizzled out. Although there have been many recent efforts to collect and maintain large repositories of these types of data, there have been relatively few studies to identify the ways these datasets could be related and linked for prognosis and maintenance decision making.

After identifying what and how to predict incipient failures and developing a corresponding prognosis, maintenance engineers must consider how to communicate the 
prediction. In this activity once again, technicians' psychosocial attributes and values may influence how they discuss prognoses with asset managers. Regardless of whether prognostic assessments are subjective or objective, however, technicians should consider two major points. Firstly, the maintenance crew should clarify in their own minds the link, if any, between their prognostic assessment and their consequent decision making. Secondly, they should consider the ways that they and their assets might benefit from explicitly discussing how the prognostic assessment is linked with diagnostics and preventive maintenance recommendations. These and other steps that maintenance engineers should take in incorporating prognostic information into their decision making are discussed in this paper. The objective is to give an overview of how the integration of disparate data sources, commonly available in industry, can be achieved for maintenance prognosis and optimal decision making.

\section{Introduction}

Probabilistic modelling of machine life and other nonparametric reliability methods developed over the past five decades mainly focus on age, and not condition, as a predictor of remaining life. Now that new sensor technologies offer means to track condition as well as age, better estimates of residual life can be made. The work by Jardine et al. [1] using the Cox's proportional hazard modeling concept integrates both age and condition information into an Age Replacement decision model without differentiating between various failure modes. Neural network models for estimating residual machine life have been proposed both as a "virtual" sensing technology [2] and as a means to estimate remaining life [3]. Damage accumulation models of residual machine life describe remaining life as a function of material creep, fatigue, embrittlement, or corrosion damage propagation until some failure limit is reached. These models presuppose knowledge of a "damage limit" which is analogous to current practice of establishing "alarm" limits currently in vogue within the predictive maintenance community. Variations on the theme of damage accumulation modelling estimate residual life as a direct function of various environmental parameters by assuming that these parameters are indeed causing material creep, fatigue, embrittlement, or corrosion damage propagation.

Current industry practices for machinery condition monitoring are focused on diagnostic matters and, in terms of predicting remaining life, rely on end-users to establish their own "alarm" or "failure" levels for each deployed condition monitoring technology based on the end-user's experience or engineering judgment. Available software programs then employ simple regression models for time-to-alarm forecasting without benefit of any knowledge regarding the value of remaining life about to be discarded. To this problem, the use of prognostics in maintenance decision making can provide a solution. However, to perform a prognosis on remaining useful life of machinery, different disparate data sources, spread throughout the entire company are necessary. The objective of this paper is to present a methodology to collect, link and process these different data sources in order to assist in maintenance prognosis and decision making.

Section 2 of this paper gives a description of the most important data sources and repositories of information for asset management. The focus is on two major data sources: CMMS and CM. An architecture for the integration of CMMS and CM systems for asset management is presented. A discussion on data-driven and model- 
based prognostics is given in Section 3. Furthermore, the use of prognostic information in maintenance decision making and support is discussed. In Section 4, all earlier discussed points on prognostics, data repositories and maintenance decision support are linked by presenting a framework based on asset cloud computing. Finally, Section 5 gives the major conclusions and identifies possibilities for future research.

\section{Data sources}

The need to improve asset performance through proper diagnosis and prognosis is considerable [4]. One barrier to improvement has been the absence of a performance management solution encompassing the various divisions of operations, maintenance, and finance, for example. With each division using its own performance metrics, it is difficult for optimal decisions to be made, such as balancing reliability goals against asset utilization goals.

Many people have been chasing the "holy grail" of self-diagnostics and prognosis. Furthermore, there are many balanced scorecards and key performance indicator solutions being offered in today's market [5]. Many seem to be making similar claims including that their product will make a manufacturing process run better, faster, more efficiently, and with greater returns. However, one of the greatest challenges for effectively improving plant asset performance is that the necessary information is scattered across disconnected silos of data in each department. Furthermore, it is difficult to integrate these silos due to several fundamental differences. For example, control system data is real-time data measured in terms of seconds, whereas maintenance cycle data is generally measured in terms of calendar based maintenance time (e.g., days, weeks, months, quarters, semi -annual, annual), and financial cycle data is measured in terms of fiscal periods.

CMMS and CM are the most popular repositories of information in maintenance, where most of the deployed technology is installed and unfortunately isolated information islands are usually created [6]. While using a good version of either technology can assist in reaching the defined maintenance goals, combining the two (CMMS and CM) into one seamless system can have exponentially more positive effects on maintenance and asset performance than either system alone might achieve. The combination of the strengths of a top-notch CMMS (preventive maintenance (PM) scheduling, automatic work order generation, maintenance inventory control, and data integrity) with the capabilities of a leading-edge CM system (multiple-method condition monitoring, trend tracking, and expert system diagnoses) in such a way that work orders are generated automatically based on information provided by CM diagnostic and prognostic capabilities. Just a few years ago, linking CMMS and CM technology was mostly a vision easily dismissed as infeasible or at best too expensive and difficult to warrant much investigation. Now, the available technology in CMMS and CM have made it possible to achieve such a link relatively easily and inexpensively (Figure 1). A topshelf CMMS can perform a wide variety of functions to improve maintenance performance. It is the central organizational tool for World-Class Maintenance (WCM). Among many other critical features, a CMMS is primarily designed to facilitate a shift in emphasis from reactive to preventive maintenance. It achieves this shift by allowing a maintenance professional to set up automatic PM work order generation. A CMMS can 
also provide historical information which is then used to adjust PM system setup over time to minimize unnecessary or redundant maintenance actions or repairs, while still avoiding run-to-failure repairs. PMs for a given piece of equipment can be set up on a calendar schedule or a usage schedule that utilizes meter readings. A fully-featured CMMS also includes inventory tracking, workforce management, purchasing, in a package that stresses database integrity to safeguard vital information. The final result is optimized equipment up-time, lower maintenance costs, and better overall plant efficiency.

On the other hand, a CM system should accurately monitor real-time equipment performance, and alert the maintenance professional to any changes in performance trends. There are a variety of measurements that a CM package might be able to track including vibration, oil condition, temperature, operating and static motor characteristics, pump flow, and pressure output. These measurements are squeezed out of equipment by monitoring tools like ferrographic wear particle analysis, proximity probes, triaxial vibration sensors, accelerometers, lasers, and multichannel spectrum analyzers. The very best CM systems are expert systems that can analyze measurements like vibration and diagnose machine faults. Expert system analysis like this puts maintenance procedures on hold until absolutely necessary, thus extracting maximum equipment up-time. In addition, the best expert systems offer diagnostic fault trending where individual machine fault severity can be observed over time.

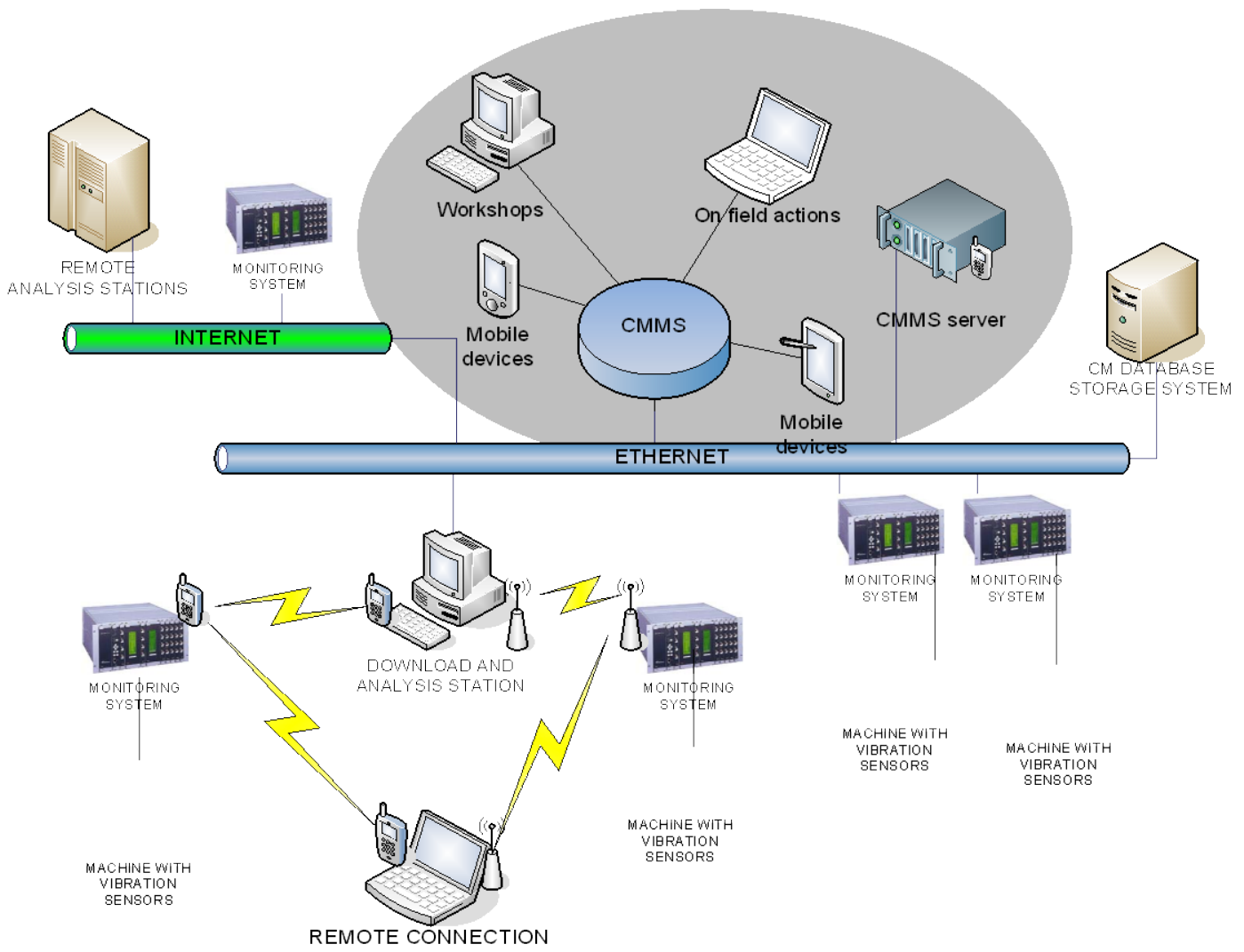

Figure 1. ICT architecture for the integration of CMMS and CM systems in maintenance and asset management. 
Both CMMS and CM systems have strong advantages that make them indispensable to maintenance operation improvements. CMMS is a great organizational tool, but cannot directly monitor equipment conditions. A CM system excels at monitoring those equipment conditions, but is not suited to organizing your overall maintenance operation. The logical conclusion, then, is to combine the two technologies into a seamless system that avoids catastrophic breakdowns, but eliminates needless repairs to equipment that is running satisfactorily.

The general opinion among the maintenance staff is that the application of information technology brings dramatic results in machine reliability and maintenance process efficiency. However, few maintenance managers can show or calculate the benefits of the application of information technologies. Technology providers are trying to develop more advanced tools while the maintenance departments seem to struggle with daily problems of implementing, integrating and operating such systems. The technology providers or the users do generally not know the feasibility of applying these technologies, but apparently they seem to improve the efficiency of the maintenance activities. The users combine their experience and heuristics in defining maintenance policies and in usage of condition monitoring systems. The resulting maintenance systems seem to be a heterogeneous combination of methods and systems in which the integrating factor of the information and business processes is the maintenance personnel. The information in the maintenance systems goes through these human minds forming an organisational information system and creating a high reliance on the expertise of the maintenance staff. Literature provides many models for maintenance decision support, however most of these models are too simple to represent real life cases conveniently [7]. Therefore, these models are definitely not widespread in industry.

With emergence of intelligent sensors to measure and monitor the health state of the component and gradual implementation of information and communication technologies (ICT) in organizations, conceptualization and implementation of e-maintenance is turning into a reality [4]. While e-maintenance shows a lot of promise, seamless integration of information and communication technologies (ICT) into the industrial environment remains a challenge. It is very critical to understand and address the requirements and constraints from the maintenance as well as the ICT standpoints in parallel.

\section{Prognostic information in DSS}

Many industrial systems exhibit increasing wear and tear of equipment during operation. Moreover, many companies want to extend the lifetime of their assets due to the increased competition and economical crisis. Prognostics are viewed as an add-on capability to diagnosis; they assess the current health of a system and predict its remaining life based on features that capture the gradual degradation in the operational capabilities of a system. Prognostics are critical to improve safety, plan successful missions, schedule maintenance, reduce maintenance cost and downtime. Unlike fault diagnosis, prognosis is a relatively new area and is becoming an important part of Condition-based Maintenance (CBM) of systems. 
Currently, there are many prognostic techniques; their usage must be tuned for each application. The prognostic methods can be classified as being associated with one or more of the following two approaches: data-driven and model-based prognostics. Each of these approaches has its own advantages and disadvantages and, consequently they are often used in combination in many applications. The following overview of prognostic techniques and their applications is provided in the context of the advantageous systems and methods disclosed.

\subsection{Data-Driven Prognostics}

The data-driven approaches are derived directly from routinely monitored system operating data (e.g. power, vibration and acoustic signal, temperature, pressure, oil debris, currents, voltages). In many applications, measured input/output data is the major source for gaining a deeper understanding of the system degradation behavior. The data-driven approaches rely on the assumption that the statistical characteristics of data are relatively unchanged unless a malfunctioning event occurs in the system. That is, the common cause variations are entirely due to uncertainties and random noise, whereas special cause variations (e.g., due to degradations) account for data variations not attributed to common causes.

The data-driven approaches are based on statistical and learning techniques from the theory of pattern recognition. These range from multivariate statistical methods (e.g., static and dynamic principle components analysis (PCA), to black-box methods based on neural networks (e.g., probabilistic neural networks (PNN), decision trees, multilayer perceptrons, radial basis functions and learning vector quantization (LVQ)), graphical models (Bayesian networks, hidden Markov models), self-organizing feature maps, signal analysis (filters, auto-regressive models, FFT, etc.) and fuzzy rule-based systems.

The research on data-driven approaches has focused on monitoring of signals related to system health. The strength of datadriven techniques is their ability to transform highdimensional noisy data into lower dimensional information for diagnostic/prognostic decisions. The main drawback of data-driven approaches is that their efficacy is highlydependent on the quantity and quality of system operational data. The data-driven approach is applicable to systems, where an understanding of first principles of system operation is not comprehensive.

\subsection{Model-Based Prognostics}

The model-based methods generally assume that an accurate mathematical model of the degradation process is available. The model-based methods use residuals as features, where the residuals are the outcomes of consistency checks between the sensed measurements of a real system and the outputs of a mathematical model. The premise is that the residuals are large in the presence of malfunctions, and small in the presence of normal disturbances, noise and modeling errors. Statistical techniques are used to define thresholds to detect the presence of faults. The three main ways of generating the residuals are based on parameter estimation, observers (e.g. Kalman filters) and parity relations. The model-based approach is generally applicable in situations where accurate 
mathematical models can be constructed from first principles. The main advantage of the model-based prognostics approach is the ability to incorporate physical understanding of the system into the monitoring of the machinery. Another advantage is that, in many situations, the changes in feature vectors are closely related to model parameters. Therefore, it can also establish a functional mapping between the drifting parameters and the selected prognostic features. Moreover, if understanding of the system degradation improves, the model can be adapted to increase its accuracy and to address subtle performance problems. Consequently, it can significantly outperform data-driven approaches. Accordingly, advantageous model-based prognostic techniques are disclosed by combining singular perturbation methods of control theory, coupled with dynamic state estimation techniques for damage prediction.

A hybrid model could combine some or all of the available model types (data-driven, and phenomenological), so that more complete information allows for more accurate recognition of the fault state. While most models incorporate some prior knowledge, little work has been done on explicit hybrid modeling for fault diagnostics and maintenance decision making. Gaps remain in understanding the overall relationships between production and reliability for systems that vary with time.

One adtional challenge is the integration of descriptive information contained in maintenance work orders and reports. For this purpose, symbolic and linguistic models can be useful. They use empirical relationships described in words rather than as mathematical or statistical relationships. For example, a semantic description may be a rule for determining whether a fault exists under a set of conditions reported in a work order. These models are good for general descriptions of causal relationships, but verbal descriptions are not effective for detailed descriptions of complicated dependencies and timevarying behaviour.

The goal for system reliability (indeed, any classification exercise) is to minimize Bayes risk, that is, to choose the lowest risk option based on the observed system outputs and conditional probabilities of what state the system is in, given the observed data. Minimum Bayes Risk decision making relies on conditional probabilities, which rely on a posteriori probabilities and prior probabilities of states of the system (in this case, fault modes). Since risk to the operation includes not only production loss but also safety hazards and environmental impacts, research is required to develop risk expressions that include these considerations in maintenance decision making.

\subsection{Use of prognostics in maintenance decision making}

Unlike conventional maintenance strategies, prognostic techniques predict system degradation based on observed system condition to support "just-in-time" maintenance. The ever increasing usage of model-based design technologies facilitates the integration of model-based diagnosis and prognosis of systems, leading to condition-based or prognostic maintenance. Condition-based maintenance is a well studied field in maintenance management. Many models in literature indicate that a condition-based maintenance policy is capable of reducing cost, increasing productivity and maintaining high equipment reliability and availability while at the same time ensuring a higher safety level. Marseguerra et al. [8] uses Monte Carlo simulation and genetic algorithms 
to determine the optimal degradation level beyond which a preventive maintenance intervention should be taken by optimizing profit and availability. A multi-component simulation modeling approach is taken by Barata et al. [9] to find the optimal degradation threshold for performing preventive maintenance actions. Liao et al. [10] introduces a condition-based availability limit policy which achieves the maximum availability of a system by optimally scheduling maintenance actions. Other papers not only try to find the optimal degradation threshold, but at the same time optimize the inspection schedule or policy [11]. Although condition-based maintenance takes advantage of the known state of components, setting a degradation threshold beyond which preventive maintenance is carried out is not always an optimal solution compared to prognostic maintenance. Prognostic maintenance uses current and predictive information like the remaining useful lifetime of components to optimally schedule maintenance actions, while condition-based maintenance only uses current component state information. The benefit of also using information about future degradation over only using currently observed information is illustrated in different publications [12-15]. Proactive maintenance decisions can be made based on the prognostic information which results in a dynamic maintenance schedule with lower costs and higher uptime of the machinery.

Two major challenges are presented in the context of prognosis and maintenance decision making. First relates to methods and systems for a prognostic assessment itself, secondly the management of this information to adopt the proper decision.

\section{Link between prognostic information in DSS and data sources}

Although there are a variety of systems and methods for monitoring and maintaining machinery and equipment, each has one or more inherent limitations which limit its usefulness. Prognosis algorithms (Section 3.1 and 3.2) operate by continuously comparing newly extracted features - i.e., machine conditions - to their corresponding baseline values. These baseline characteristics are essentially the statistical means of the features collected during the setup phase and decisions are taken based on their evolution. The prognosis capabilities of conventional predictive maintenance systems are based on applying different types of thresholds, templates, and rules, to quantify the relationship between the current feature values and their baseline counterparts.

One limitation of this type of systems is that during the process of monitoring the machine features, the thresholds remain unchanged which is also a major assumption in most of the publications on maintenance optimization and decision making based on condition monitoring and prognostic information (Section 3.3). The only time when these thresholds are reconsidered is when an expert interferes to force their recalculation. This type of human intervention usually results from the observation of frequent false alarms caused by a process mean shift. Therefore, it would be desirable to have a method for predictive maintenance of a machine which utilizes unsupervised learning techniques, and which can identify a significant change in the pattern of monitored machine feaures. This dynamic benchmarking [16] is essential to take proper decisions based on the real age and condition of the machine and not just on the set-up values of its initial deployment. 
Another limitation of conventional machine monitoring methods and condition-based maintenance (CBM) technologies is that their application is limited to a particular machine. Over time, there may be extensive characterization of the physical and mechanical principles that guide the equipment behavior and evolution. While this may lead to accurate information about a particular machine, such technologies are extremely limiting when it comes to widespread deployment for a wide variety of equipment. Therefore, it would be desirable to have a method for predictive maintenance of a machine, which developed a "generic" framework that was relatively independent of the type of physical equipment under consideration.

This framework has to be based on a common set of data repositories and knowledge extraction tools. Cloud computing seems to be a feasible solution for integration purposes in this stage. The cloud in cloud computing provides the means through which everything from computing power to computing infrastructure, applications, business processes to personal collaboration can be delivered as a service wherever and whenever it is needed. The cloud itself is a set of hardware, networks, storage, services, and interfaces that enable the delivery of computing as a service. For asset management, the cloud seems to be the solution with such amounts of dispersed data in different repositories. The end user (maintenance or operators) do not really have to know anything about the underlying technology. The data collection and distribution applications may be dispersed throughout the network and collection of data may be accomplished at distributed locations. The collected data may then be converted to a common format at the distributed locations and sent to one or more central databases for subsequent distribution. These distributed databases will constitute the asset cloud.

The data collection and distribution system, cloud, will collect the data from the different data sources in a common format or will convert that data, once received, to a common format for storage and use later by other elements, devices or applications in the process control system [17]. Once received and converted, the data is stored in a database in some accessible manner and is made available to applications or users within the asset cloud. Applications related to process control, alarming, device maintenance, fault diagnostics, predictive maintenance, financial planning, optimization, etc. may use, combine and integrate the data from one or more of the different data sources to operate better than these applications have been able to operate in the past without data from vastly different or previously inaccessible data sources.

A more detailed data flow diagram illustrating data flow within the process control plant is provided in Figure 2. Beginning at the left side of the diagram, data associated with the process plant is collected by or at different functional areas or data sources within the plant:

- Control data is collected by, for example, typical process control devices such as field devices, input/output devices, handheld or remote transmitters, or any other devices which may be, for example, communicatively connected to process controllers.

- Equipment monitoring data associated with traditional equipment monitoring activities is collected by, for example, sensors, devices, transmitters, or any other devices within the plant. Process performance data may be collected by the same or other devices. 
- Financial data may be collected by other applications run in computers in the process control plant as part of the performance monitoring data

- Collected data may be from applications or sources outside of the traditional process control network, such as applications owned and operated by service organizations or vendors.

This data may be collected in any manner including automatically or manually since many diverse sources can be used to monitor equipments. Thus, data collectors may include hand held collection devices, laboratory chemical and physical measurements, fixed or temporary on-line devices, devices which periodically (e.g., RF) telemeter data from remote process and equipment measurement devices, on-line device inputs or remote multiplexers and/or concentrators or any other data collection devices.

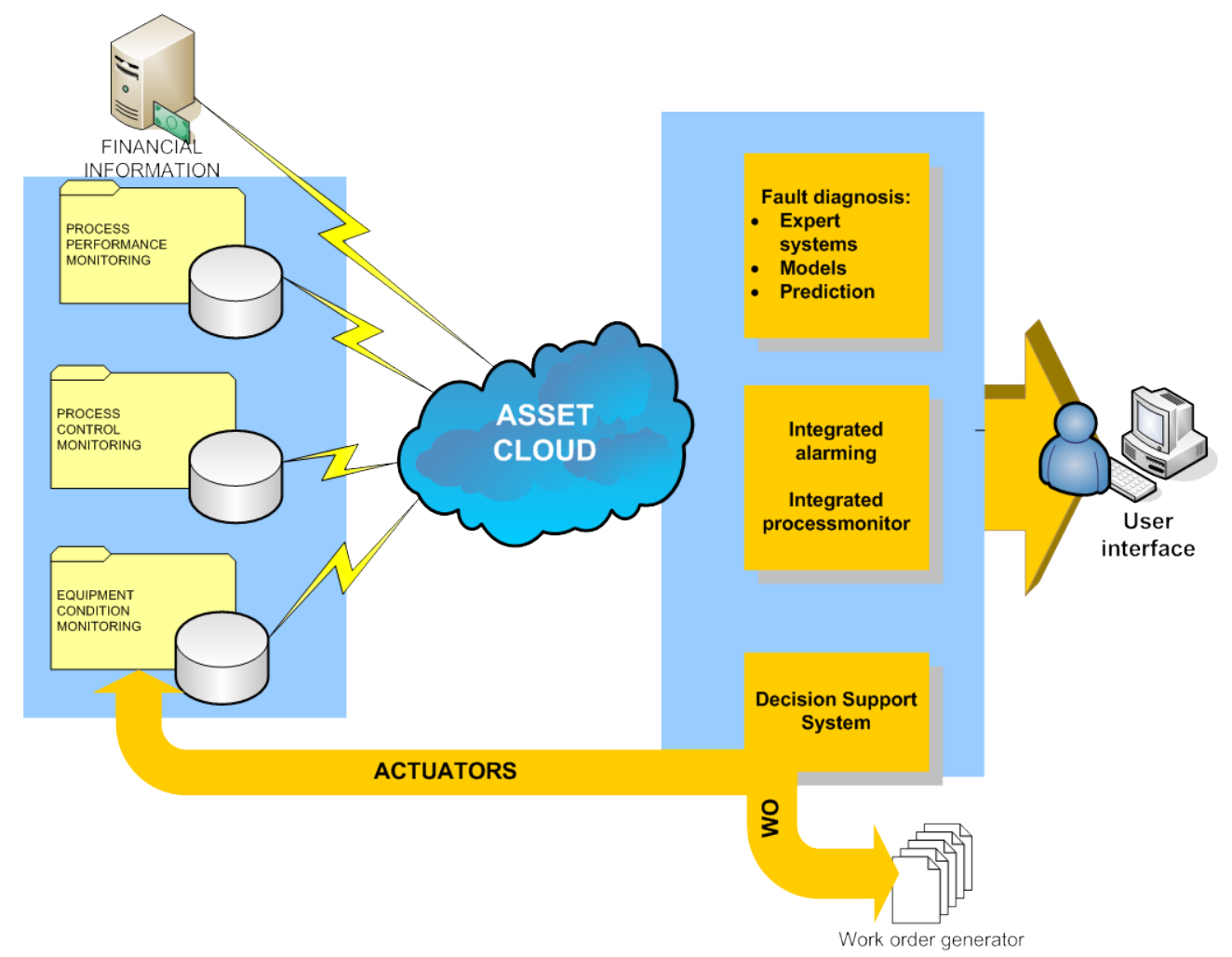

Figure 2. Data flow diagram of info sources in process control.

The process control data, equipment monitoring data and process performance data may be reconciled, verified, validated and/or formatted by data collection and reconciliation applications (which may be part of the cloud) run within the data collection device or within any other device such as at a central data historian, process controllers, equipment monitoring applications, etc. or any other device which receives or processes this data. After being reconciled in any known or desired manner or, in some cases, not being reconciled at all, the collected data may be provided to one or more applications typically associated with the different functional areas of the process control system. Further, one or more diagnostic applications may use the collected process control data to perform process control diagnostics. Such diagnostic applications may include, for example, applications which help an operator pinpoint problems within process control loops, instruments, actuators, etc. The diagnostic applications may also include expert diagnostic engines. Of course, the process diagnostic applications can take the form of 
any other typical or standard process diagnostic applications and are not limited to these specifically mentioned herein. The outputs of these diagnostic applications can take any form and may, for example, indicate faulty or poorly performing loops, function blocks, areas, units, etc. within the process control system.

The equipment monitoring functional block will receive the equipment condition data. The equipment monitoring functional block may include equipment or condition monitoring applications which may, for example, accept or generate alarms indicating problems with various pieces of equipment detect poorly performing or faulty equipment within the plant or detect other equipment problems or conditions which may be of interest to a maintenance person. Equipment monitoring applications are well known and typically include utilities adapted to the different specific types of equipment within a plant. Likewise, equipment diagnostic applications may be implemented to detect and diagnose equipment problems based on raw data measured pertaining to the equipment. Such equipment diagnostic applications may include, for example, vibration sensor applications, rotating equipment applications, power measurement applications, etc. Of course, there are many different types of known equipment condition monitoring and diagnostic applications which can produce many kinds of different types of data associated with the state or operating condition of different pieces of equipment within a process control plant. Still further, a historian may store raw data detected by equipment monitoring devices, may store data generated by the equipment condition monitoring and diagnostic applications and may provide data to those applications as needed. Likewise, equipment models may be provided and used by the equipment condition monitoring and diagnostic applications and in any desired manner.

\section{Conclusions and future work}

This paper discusses how disparate data sources (i.e. CMMS and CM), commonly available in industry, can be integrated to perform maintenance prognosis and optimal maintenance decision making. An architecture for the integration of CMMS and CM systems in asset management is presented. Furthermore, the link with prognostic approaches and corresponding decision support tools is made. Cloud computing is believed to be a feasible solution for the integration of these disparate data sources, prognostics and maintenance decision support. Future work will be on the further development of a general framework for the integration of disparate data sources to perform maintenance prognosis and maintenance decision making.

\section{Acknowledgments}

This work has been carried out within the framework of the Prognostics for Optimal Maintenance (POM2) project (grant nr. 100031), financially supported by the Institute for the Promotion of Innovation through Science and Technology in Flanders.

\section{References}

1. Jardine, A.K.S., T. Joseph, and D. Banjevic, 'Optimizing condition-based maintenance decisions for equipment subject to vibration monitoring', Journal of Quality in Maintenance Engineering, Vol 5, No 3, pp 192-202, 1999. 
2. Upadhyaya, B.R. and E. Eryurek, 'Application of neural networks for sensor validation and plant monitoring', Nuclear Technology, Vol 97, No 2, pp 170176, 1992.

3. Shao, Y. and K. Nezu, 'Prognosis of remaining bearing life using neural networks', Proceedings of the Institution of Mechanical Engineers. Part I, Journal of systems and control engineering, Vol 214, No 3, pp 217-230, 2005.

4. Muller, A., A. Crespo Marquez, and B. Iung, 'On the concept of e-maintenance: Review and current research', Reliability Engineering \& System Safety, Vol 93, No 8, pp 1165-1187, 2008.

5. Kaplan, R.S. and D.P. Norton, 'The balanced scorecard-measures that drive performance', Harvard Business Review, Vol 70, No 1, pp 71-79, 1992.

6. Galar, D., et al., 'Maintenance decision making based on different types of data fusion', Eksploatacja i niezawodnosc - Maintenance and Reliability, Vol 14, No 2, pp 135-144, 2012.

7. Van Horenbeek, A., L. Pintelon, and P. Muchiri, 'Maintenance optimization models and criteria', International Journal of Systems Assurance Engineering and Management, Vol 1, No 3, pp 189-200, 2010.

8. Marseguerra, M., E. Zio, and L. Podofillini, 'Condition-based maintenance optimization by means of genetic algorithms and Monte Carlo simulation', Reliability Engineering \& System Safety, Vol 77, No 2, pp 151-165, 2002.

9. Barata, J., et al., 'Simulation modelling of repairable multi-component deteriorating systems for [']on condition' maintenance optimisation', Reliability Engineering \& System Safety, Vol 76, No 3, pp 255-264, 2002.

10. Liao, H., E.A. Elsayed, and L.-Y. Chan, 'Maintenance of continuously monitored degrading systems', European Journal of Operational Research, Vol 175, No 2, pp 821-835, 2006.

11. Grall, A., et al., 'Continuous-time predictive-maintenance scheduling for a deteriorating system', Reliability, IEEE Transactions on, Vol 51, No 2, pp. 141150, 2002.

12. Camci, F., 'System Maintenance Scheduling With Prognostics Information Using Genetic Algorithm', IEEE Transactions on Reliability, Vol 58, No 3, pp 539552, 2009.

13. Yang, Z.M., D. Djurdjanovic, and J. Ni, 'Maintenance scheduling in manufacturing systems based on predicted machine degradation', Journal of intelligent manufacturing, Vol 19, No 1, pp 87-98, 2008.

14. Bouvard, K., et al., 'Condition-based dynamic maintenance operations planning \& grouping. Application to commercial heavy vehicles', Reliability Engineering \& System Safety, Vol 96, No 6, pp 601-610, 2011.

15. Van Horenbeek, A. and L. Pintelon. 'Optimal prognostic maintenance planning for multi-component systems' Proceedings of the European Safety and Reliability Conference: ESREL 2011, Troyes, France: CRC Press/Balkema, 2011.

16. Galar, D., L. Pilar, and B. Luis, 'Application of dynamic benchmarking of rotating machinery for e-maintenance', International Journal of Systems Assurance Engineering and Management, Vol 1, No 3, pp 246-262, 2010.

17. Galar, D. and A. Morant, 'Integration of Production Data in CM for Nonstationary Machinery: A Data Fusion Approach', Proceedings of the second international conference CMMNO’2012, Springer: Tunisia. pp. 403-414, 2012. 